\title{
INHALTSVERZEICHNIS - TABLE DES MATIĖRES - TABLE OF CONTENTS - ÍNDICE - INDICE
}

JOSE MIGUEL DE BARANDIARÁN, Navarra. La cueva de Ekain, Guipúzcoa, España

Seite

1

MARTIN ALMAGRO GORBEA, Madrid. La cueva del Niño (Ayna, Prov. Albacete, España) un yacimiento con representaciones de arte rupestre de estilo paleolitico

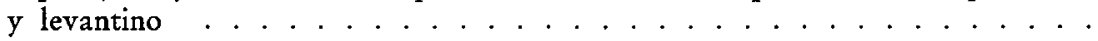

GERHARD RICHTER, Krefeld. Die Kunstwerke der Kniegrotte von Döbritz bei Pössneck . . . . . . . . . . . . . . . . . . . . .

UWE TOPPER, Berlin. Felsbilder der Kanarischen Inseln . . . . . . . . . . . . . . 29

MUVAFFAK UYANIK, Istanbul. Die Felszeichnungen in der Türkei . . . . . . . . . 35

OTTO-HERMAN FREY, Hamburg. Die Kunst der Situlen. Metallarbeiten der Vorzeit im Südost-Alpenraum . . . . . . . . . . . . . . . . . . . . .

DOROTHEE RENNER, Mainz. Die runden, durchbrochenen Zierscheiben der Merowingerzeit ..................... . . . . 46

HERBERT KÜHN, Mainz. Die Christus-Schnallen der Völkerwanderungszeit . . . . . 51

GYULA LÁSZLO, Budapest. Darstellung des sakralen Zweikampfes auf einer awarischen Knochenschnitzerei

PAVEL CERVICEK, Frankfurt a. M. Datierung der nordafrikanischen Felsbilder durch die Patina............................. 82

HARALD PAGER, Johannesburg. An experiment with film animation . . . . . . . 88

GERHARD J. FOCK, Kimberley. Südafrikanische Felsgravierungen. Ein Überblick . . . 90

A. P. OKLADNIKOW, Nowosibirsk. Die Felsbilder am Amur und am Ussuri . . . . . 95

ANDREAS LOMMEL, München. Felsbilder in Australien . . . . . . . . . . . 106

CHEWON KIM, Seoul. Studies of Dolmens in Korea . . . . . . . . . . . . . . . . 112

CARL HENTZE, Darmstadt. Antithetische T’ao-t'ieh-Motive . . . . . . . . . . . . 118

MITTEILUNGEN - COMMUNICATIONS - COMMUNICACIONES COMMUNICACIONI

VON HERBERT KƯHN

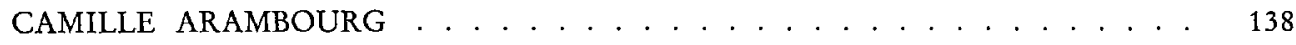

MILES CRAWFORD BURKITT . . . . . . . . . . . . . . 138 
Seite

TH. McCOWN .............................. 138

LEO FROBENIUS . . . . . . . . . . . . . . . . . . . . . . . . . . . 138

ALBERT EGGES VAN GIFFEN . . . . . . . . . . . . . . . . . . 139

FERDINAND KUTSCH . . . . . . . . . . . . . . . . . . 139

JULIO MARTINEZ SANTA-OLALLA . . . . . . . . . . . . . . . . 139

WILHELM UNVERZAGT . . . . . . . . . . . . . . . . . . 140

Der Stand der Forschung über die Kunst der Eiszeit . . . . . . . . . . . . . . . . . 140

Die Deutung der Kunst der Eiszeit . . . . . . . . . . . . . . . . . . . 143

Die Datierung der Kunst der Eiszeit . . . . . . . . . . . . . . . 150

Die Statuette aus Monpazier, Dordogne . . . . . . . . . . . . . . . . 152

Eine Männliche Statuette aus Hohlenstein-Stadel, Baden-Württemberg . . . . . . . . . 153

Les Fouilles de Gönnersdorf . . . . . . . . . . . . . . . . . . . . . . . . . . . 154

Die Ausgrabung von Thera und das Problem Atlantis . . . . . . . . . . . . . . . . 154

Funde aus der Quelle der Seine . . . . . . . . . . . . . . . . . 156

BESPRECHUNGEN - COMPTES RENDUS - REVIEWS

CRITICAS - CRITICHE

VON HERBERT KÜHN

L. PERICOT-Garcia u. a., Prehistoric and Primitive Art . . . . . . . . . . . 159

ANDRE LEROI-GOURHAN, Prähistorische Kunst . . . . . . . . . . . . . . . 160

HERBERT KUHN, Die Felsbilder Europas . . . . . . . . . . . . . . 161

MARIE E. P. KONIG, Am Anfang der Kultur . . . . . . . . . . . . . . . . . . . 162

HERMANN MULLER-KARPE, Handbuch der Vorgeschichte. 2. Band . . . . . . . . 163

JAN FILIP u. a., Enzyklopädisches Handbuch, Bd. II . . . . . . . . . . . . . . . . 164

H. JELINEK, Das große Bilderlexikon des Menschen in der Vorzeit . . . . . . . . 164

MICHEL BREZILLON, Dictionaire de la Préhistoire . . . . . . . . . . . . . . . 165

EMMANUEL ANATI u. a., Valcamonica Symposium . . . . . . . . . . . . . . 165

Mélanges de Préhistoire, d'Archéocivilisation et d'Ethnologie, offerts à Varagnac . . . . 167

Führer zu vor- und frühgeschichtlichen Denkmälern . . . . . . . . . . . . 168

ANDRE VARAGNAC, $\mathrm{La}$ conquête des énergies . . . . . . . . . . . . . . . . 168

GERHARD HEBERER, Homo - unsere Ab- und Zukunft . . . . . . . . . . . . . 169

MICHAIL MICHAILOWITSCH GERASSIMOW, Ich suchte Gesichter . . . . . . . . 170 
Seire

HERBERT WENDT, Der Affe steht auf . . . . . . . . . . . . . 170

RICHARD FESTER, Die Eiszeit war ganz anders . . . . . . . . . . . . . . . . . . 170

HANS KOEPF, Zauber der Archäologie . . . . . . . . . . . . . . . . . 171

JOACHIM REHORK, Faszinierende Funde, Archäologie heute . . . . . . . . . . . 171

EVA LORENZ, Grabräuber, Raubgräber, Archäologen . . . . . . . . . . . . . . , 172

HERMANN MULLER-KARPE, Das vorgeschichtlidhe Europa . . . . . . . . . . . 173

STUART PIGGOT, Vorgeschichte Europas . . . . . . . . . . . . . . 175

LEONHARD FRANZ, Die Kultur der Urzeit Europas . . . . . . . . . . . . . . 175

ANDRE DE CAYEUX u. a., La France au temps des Mammouths . . . . . . . . . . 176

DENISE DE SONNEVILlE-BORDES, La Préhistoire Moderne . . . . . . . . . . . 176

A. LEMOZI u. a., Pech Merle. Le Combel. Marcenac . . . . . . . . . . . . . . . . 177

ANTONIO BELTRÁN u. a., La Cueva de Bédeilhac . . . . . . . . . . . . . . . . 177

MAX SARRADET, Font-de Gaume en Périgord . . . . . . . . . . . . . . . 177

BENITO MADARIAGA DE LA CAMPA, Las pinturas rupestres . . . . . . . . . . 178

LEON PALES, Les Gravures de La Marche, Bd. I . . . . . . . . . . . . . . . . . . 178

MICHEL ROUSSEAU, Les grands félins dans l'art de notre Préhistoire . . . . . . . 179

ANTONIO BELTRĀN MARTINEZ, Arte Rupestre Levantino . . . . . . . . . . . 179

EDUARDO RIPOLL-PERELLO, The painted shelters, Santolea, Teruel . . . . . . . 179

ANTONIO BELTRÁN MARTINEZ, Los abrigos pintados de la Cañaica . . . . . . . 180

ANDERS HAGEN, Studier i vestnorsk bergkunst . . . . . . . . . . . . . . 180

ANTONIO BELTRÁN MARTINEZ, Las pinturas esquematiquas de Lecina . . . . . 180

VLADIMIR DUMITRESCU, L'Art néolithique en Roumanie . . . . . . . . . . . . 181

VLADIMIR DUMITRESCU, L'arte preistorica in Romania . . . . . . . . . . . . . 181

ERNST BURGSTALLER, Felsbilder in Osterreich . . . . . . . . . . . . . 181

EMMANUEL ANATI, I pugnali nell' arte rupestre . . . . . . . . . . . . . . 182

P. V. GLOB, Helleristninger i Danmark . . . . . . . . . . . . . . . . . 183

WALTER MAIER-ARENDT, Die Bandkeramische Kultur im Untermaingebiet . . . . 183

ROLF MULLER, Der Himmel über dem Menschen der Steinzeit . . . . . . . . . . . 184

ROLF HACHMANN, Die Germanen . . . . . . . . . . . . . . . . . 185

GERHARD MILDENBERGER, Sozial- und Kulturgeschichte der Germanen . . . . . 185

JEAN JAQUES HATT, Kelten und Galloromanen . . . . . . . . . . . . . . . . . 186

WILLY HARTNER, Die Goldhörner von Gallehus . . . . . . . . . . . . . . . . . 187

RAYMOND BLOCH, Die Etrusker . . . . . . . . . . . . . . . . . . . 189

J. V. LUCE, Atlantis, Legende und Wirklichkeit . . . . . . . . . . . . . . 190 
Seite

HEINZ LUCAS, Der Tanz der Kraniche . . . . . . . . . . . . . . . . . . . . . 191

ERNST MEYER, Heinrich Schliemann, Kaufmann und Forscher . . . . . . . . . . . 191

BARRY CUNLIFFE, Fishbourne. Rom in Britannien . . . . . . . . . . . . . . . . 192

FRITZ JENO, Gorsium . . . . . . . . . . . . . . . . . 193

ARTHUR SUHLE, Die Münze . . . . . . . . . . . . . . . . . 194

CHRISTOF BOEHRINGER, Zur Chronologie mittelhellenistischer Münzserien . . . . 194

JOSEF REITINGER, Oberösterreich in ur- und frühgeschichtlicher Zeit . . . . . . . . 195

WILHELM ANGELI u. a., Krieger und Salzherren . . . . . . . . . . . . . . . . . 196

OTTO-HERMAN FREY, Die Entstehung der Situlenkunst . . . . . . . . . . . . . 196

HERMANN DANNHEIMER und ROMAN FINK, Fundort Bayern . . . . . . . 197

CONSTANTIN DAICOVICIU und EMIL CONDURACHI, Rumänien . . . . . . . 198

Reallexikon der Germanischen Altertumskunde, 2. Auflage . . . . . . . . . . . . . . 199

JEAN HUBERT u. a., Frühzeit des Mittelalters . . . . . . . . . . . . . . . . . . . 199

FRIEDRICH GARSCHA, Die Alamannen in Südbaden . . . . . . . . . . . . . 199

URSULA KOCH, Die Grabfunde der Merowingerzeit aus dem Donautal . . . . . . . 200

PETER LA BAUME, Das fränkische Gräberfeld von Junkersdorf . . . . . . . . . . 200

CHRISTIANE NEUFFER-MƯLLER, Ein Reihengräberfriedhof, Sontheim . . . . . . 200

CHRISTIANE NEUFFER-MULLER, Das fränkische Gräberfeld, Iversheim . . . . . 201

HERMANN AMENT, Fränkische Adelsgräber von Flonheim . . . . . . . . . . . . 201

RAINER CHRISTLEIN, Das alamannische Gräberfeld, Dirlewang . . . . . . . . . 202

BERTHOLD SCHMIDT, Die späte Völkerwanderungszeit in Mitteldeutschland . . . . 202

RUDOLF MOOSBRUGGER-LEU, Die Schweiz zur Merowingerzeit . . . . . . . . . 202

EDUARD BENINGER u. HERBERT MITSCHA-MARHEIM, Das langobardische

Gräberfeld von Nikitsch . . . . . . . . . . . . . . 203

OTTO VON HESSEN, Die langobardischen Funde von Testona . . . . . . . . . . . 204

JOSEPH DECAENS, Un nouveau cimetière, Hérouvillette . . . . . . . . . . . . . 204

G. DE BOE, Een merovingisch Grafveld te Borsbeek . . . . . . . . . . . . . . . . . 204

RAYMOND BRULET, Répertoires archéologiques de Charleroi . . . . . . . . . 205

G. FAIDER-FEYTMANS, Les Collections d'Archéologie du Musée de Mariemont . . . 205

BEDRICH SVOBODA, Cechy V Době Stehování Národů . . . . . . . . . . . . . 206

AGNES SALOMON u. a., Das völkerwanderungszeitliche Gräberfeld von Környe . . . 206

ADRIAAN VERHULST u. a., Early Medieval Studies . . . . . . . . . . . . 207

PETER CLEMOES, Anglo-Saxon England, Bd. I . . . . . . . . . . . 207

GYULA LÁSZLO, Steppenvölker und Germanen . . . . . . . . . . . . . . . . . 207 
Seite

DOROTHEE RENNER, Die durchbrochenen Zierscheiben der Merowingerzeit . . . . . 208

PETER PAULSEN und HELGA SCHACH-DORGES, Holzhandwerk der Alamannen 209

HOLGER ARBMAN, The Vikings . . . . . . . . . . . . . . . . 210

DAVID M. WILSON, The Vikings and their Origins . . . . . . . . . . . . . . 211

Reallexikon der Assyriologie und Vorderasiatischen Archäologie . . . . . . . . . . 211

E. A. S. BUTTERWORTH, The tree at the navel of the earth . . . . . . . . . . . . 212

KATHLEEN KENYON, Jerusalem. Die heilige Stadt . . . . . . . . . . . . . . . . 212

JOACHIM REHORK, Archäologie und biblisches Leben . . . . . . . . . . . . . . . 213

BURCHARD BRENTJES, Von Schanidar bis Akkad . . . . . . . . . . . . . . 214

EVELYN KLENGEL-BRANDT, Reise in das alte Babylon . . . . . . . . . . . . 215

MORTIMER WHEELER, Flammen über Persepolis . . . . . . . . . . . . 215

BRIAN DOE, Südarabien . . . . . . . . . . . . . . . . . 215

L. I. ALBAUM und B. BRENTJES, Wächter des Goldes . . . . . . . . . . . . . . . 216

JOHANNES A. H. POTRATZ, Luristanbronzen . . . . . . . . . . . . . . . . . . 217

MICHAIL GRJASNOW, Südsibirien . . . . . . . . . . . . . 218

A. P. OKIADNIKOW, Masken aus der Frühzeit am Amur . . . . . . . . . . . . . 218

A. P. OKLADNIKOW und A. M. MARTYNOW, Ein Schatz von Felsbildern am Fluß
Tom . . . . . . . . . . . . . . . . . . . . . . . . . . . . . . . . . . . . . . 219

A. P. OKLADNIKOW, Der Hirsch mit dem goldenen Geweih . . . . . . . . . . . . 219

A. P. OKLADNIKOW und W. D. SAPOROSHSKAJA, Felsbilder an der Lena . . . . 220

N. N. DIKOW, Rätselhafte Felsbilder aus Tschukotka . . . . . . . . . . . . . . . . 221

E. B. VADETSKAIA, Les idoles anciennes de l'Iénissei . . . . . . . . . . . . . 222

CHEWON KIM and MOO-BYONG YOUN, Studies of Dolmens in Korea . . . . . . 222

CYRIL ALDRED, Echnaton, Gott und Pharao Agyptens . . . . . . . . . . . 223

GILLETTE et LOUIS LEFEBVRE, Corpus des gravures de la région de Constantine 224

FRANK WILLETT, Ife, Metropole afrikanischer Kunst . . . . . . . . . . . . . . . 224

WALTHER F. E. RESCH, Die Felsbilder Nubiens . . . . . . . . . . . . . . . 225

ERNST R. SCHERZ, Felsbilder in Südwest-Afrika . . . . . . . . . . . . . . . 226

MARIA WILMAN, The Rock-Engravings of Griqualand and Bechuanaland . . . . . 227

HARALD PAGER, Ndedema. A documentation of the paintings . . . . . . . . . 227

C. W. CERAM, Der erste Amerikaner . . . . . . . . . . . . . . . . . . 228

MICHAEL D. COE, Die Maya . . . . . . . . . . . . . . . . . . . . 229

H. D. DISSELHOFF, Das Imperium der Inka . . . . . . . . . . . . . . . . 229

ROLF MƯLLER, Sonne, Mond und Sterne über dem Reich der Inka . . . . . . . . . 230

I. BOLZ-AUGENSTEIN und H. D. DISSELHOFF, Sammlung Ludwig-Aachen . . . . 231 
Seite

HANS BIEDERMANN, Altmexikos heilige Bücher ............. 231

CARLO T. E. GAY, Chalcacingo . . . . . . . . . . . . . . . . 232

CAMPBELL GRANT u. a., Rock drawings of the Coso Range . . . . . . . . . . . 232

MARIA REICHE, Geheimnis der Wüste, Peru . . . . . . . . . . . . . 233

FERNANDO A. MARBAN LAUCER, Pintura rupestre y petroglifos en San Domingo 233

MARCEL F. HOMET, Die Söhne der Sonne, Amazonas . . . . . . . . . . . . . 234

ROBERT GARDNER und KARL G. HEIDER, Dugum Dani, Neuguinea . . . . . . 234

AGNES SUSANNE SCHULZ, Felsbilder in Nord-Australien . . . . . . . . . . 235

A. R. VERBRUGGE, Corpus of the Hand Figurations . . . . . . . . . . . . . . . 236

UMSCHAU - REVUE - REVIEW

VON HERBERT KÜHN

R. ROBERT et L. R. NOUGIER, Scène d'initiation . . . . . . . . . . . . . . . . 237

L. R. NOUGIER et R. ROBERT, Pendeloque au cheval sautant . . . . . . . . . . . 237

L. R. NOUGIER et R. ROBERT, Pierres avec gravures . . . . . . . . . . . . . . 238

L. R. NOUGIER, La genèse de la «grecque * . . . . . . . . . . . . . . . 238

MARTIN ALMAGRO GORBEA, Las fechas de C14 . . . . . . . . . . . . 238

GERHARD BOSINSKI, Grabungen in Feldkirchen-Gönnersdorf . . . . . . . . . . 238

KAREL VALOCH, Kunst der Eiszeit in Mähren . . . . . . . . . . . . . . . 238

HANS-GEORG BANDI, Jungpaläolithikum in der Schweiz .......... . 238

PAUL EPPEL, Eiszeitliche Kunst in Osterreich . . . . . . . . . . . . . . . 239

M. R. LUCAS DE VIÑAS, Solapo del Aguila, Prov. Segovia . . . . . . . . . . . . 239

AKE HYENSTRAND, Slagsta in Botkyrka, Södermanland . . . . . . . . . . 239

MARTIN ALMAGRO BASCH, Los ídolos de Hernan Perez, Cáceres . . . . . . . . 239

RENATE ROLLE, Skythische und sakische Grabanlagen . . . . . . . . . . . . . 239

JEAN DEKAN, Herkunft der Bronzeindustrie des VII. Jh. . . . . . . . . . . . . . 239

N. K. SANDARS, Orient in celtic art . . . . . . . . . . . . . . . . . 240

TORSTEN CAPELLE und HAYO VIERECK, Modeln der Merowingerzeit . . . . . . 240

K. HOREDT und D. PROTASE, Das zweite Fürstengrab von Apahida . . . . . . . 240

K. HOREDT und D. PROTASE, Schatzfund aus Cluj-Someseni . . . . . . . . . . . 240

MARTIN ALMAGRO BASCH, Las representaciones de carros . . . . . . . . . . 241

PATRICIA VINNICOMBE, Motivation in African rodk art . . . . . . . . . . . 241

O. MENGHIN y C. J. GRADIN, La Piedra Calada de los Olumas . . . . . . . . . 241 